\title{
Effects of Interface Interactions on Mechanical Properties in RDX-Based PBXs HTPB-DOA: Molecular Dynamics Simulations
}

\author{
Mounir Jaidann ${ }^{1,2}$, Louis-Simon Lussier ${ }^{1}$, Amal Bouamoul ${ }^{1}$, Hakima Abou-Rachid ${ }^{1}$, \\ and Josée Brisson ${ }^{2}$ \\ ${ }^{1}$ Défense R \& D Canada-Valcartier 2459 Boul. Pie-XI Nord, Québec, QC, \\ Canada G3J 1 X5 \\ ${ }^{2}$ CERMA and CQMF, Département de chimie, Faculté des sciences et de génie, \\ Université Laval, Québec, Canada G1V 0A6 \\ \{Mounir.Jaidann, Louis-Simon.Lussier, Amal. Bouamoul, \\ Hakima.Abou-Rachid\} @drdc-rddc.gc.ca, \\ Josee.Brisson@chm.ulaval.ca
}

\begin{abstract}
Atomistic molecular dynamics simulation was carried out to study interface interactions between a crystal structure and a plastic bonded explosive (PBX) system. In this work, the polymer is hydroxyl-terminated polybutadiene (HTPB), the plasticizer is dioctyl adipate (DOA) and the crystal phase is hexahydro-1,3,5-trinitro-1,3,5-triazine (RDX). Experimental RDX crystallo-graphic data show that $(020),(200)$ and (210) crystal faces usually dominate, and these were therefore only these were studied. Interface models were built and interfacial bonding energies calculated to investigate HTPB/RDX adhesion properties in the (DOA+HTPB)/RDX system. Mechanical properties such as Poisson's ratio, Young, bulk and shear moduli were also predicted. The most favourable interactions occur between HTPB-DOA and the RDX (020) crystal face: obtaining crystals with prominent (020) faces may provide a more flexible mixture, with a lower Young's modulus and an increased ductility.
\end{abstract}

Keywords: Polymer-bonded explosives, molecular dynamics simulation, binding energy, mechanical properties.

\section{Introduction}

Plastic-bonded explosives (PBXs) are widely used in many defence and economic application because of their good safety, processing ease and high strength. The next generations of PBXs materials will possess improved insensitivity and energetic density while maintaining a good mechanical integrity. Atomistic molecular modeling may become a helpful tool in formulation conception, providing predictions on various properties of these systems. The use of modelling techniques can decrease hazards and accidents during formulation development, and contribute to minimizing the time-frame in which they can be screened and tested, eliminating poor formulations before even having to synthesize the compounds. Solid propellants and plastic bonded explosives incorporate various components, each having a specific function. First and 
foremost, a high density crystalline energetic material must be chosen, such as 1,3,5triaza-1,3,5-trinitrocyclohexane (RDX), shown in Fig. 1, which has a high storage stability and is considered as the most powerful high explosive. The energetic material is incorporated in a polymeric binder, which provides acceptable mechanical properties and therefore greatly decreases the risk of accidental ignition during transportation, handling, and storage. The ability of a solid propellant to perform as an insensitive energetic material depends heavily on both the binder-plasticizer compatibility and explosives compatibility to binder and plasticizer. A binder such as hydroxyterminated polybutadiene (HTPB) requires a plasticizer with a similar chemical structure. A low weight aliphatic ester like dioctyladipate (DOA) is often used. With RDX as the main body $(\approx 90 \%$ and above $)$, PBXs contain a small amount of polymer/plasticizer $(\approx 10 \%)$. Much attention has been paid to the explosives and polymers/plasticizer with respect to experimental measurement methods [1-6], as well as molecular dynamics (MD) simulation [7, 8]. However, few mechanical property simulations have been reported.

The HTPB-DOA system shown in Fig. 1 is a good model to test the use of modelling techniques for explosives, and has been the object of previous work in our group [9]. Crystalline surface morphology and crystal structure main planes existing at the crystal surface of RDX are known, and consist mainly in (020), (200) and (210) faces [10], depending on solvent. The present work will therefore focus on simulating the HTPB-DOA/RDX interface at these crystal faces. Simulations of crystalamorphous phase have already been reported $[7,11,12]$. No investigation has however been performed in the presence of plasticizer, which is one of the key factors in PBXs stability and formulation.

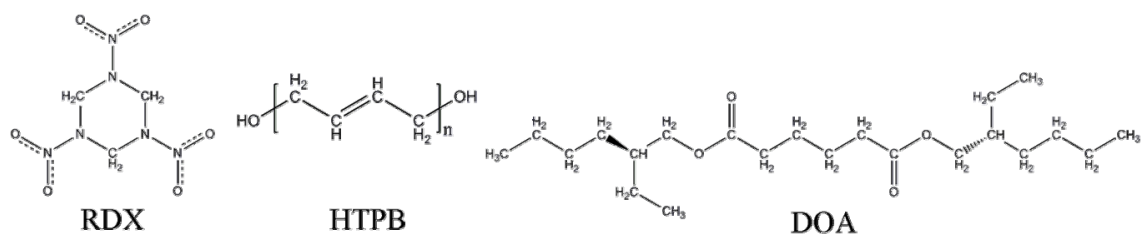

Fig. 1. Chemical structure of molecules in the studied system

Our main interest in the present work will therefore be to test the ability of atomistic methods to predict interactions between RDX and HTPB-DOA polymerplasticizer system. Mechanical properties, interface between RDX crystal surface and the HTPB-DOA blend and interfacial bonding energy will also be investigated.

The final objective is to understand fundamental properties of RDX-based PBXs, which is of utmost importance in design and development of novel energetic materials. The present work will also allow investigation of the most interesting crystal morphology for RDX, interaction wise, and may allow for a better selection of experimental conditions (solvent, temperature, etc) in its processing prior to incorporation in PBXs. This work is part of a larger effort to evaluate composite systems prior to experimentally investigation, in search for new, more optimized explosives. 


\section{Computational Details}

Molecular simulations were performed using the Materials Studio (MS) version 4.3 software commercialized by Accelrys. Molecular mechanics (MM) and molecular dynamics (MD) calculations were performed using the Discover module and the COMPASS (condensed-phase optimized molecular potentials for atomistic simulation studies) force field [13] under periodic boundary conditions. Coulombic interactions were described with the Ewald summation approach with an accuracy of $0.01 \mathrm{kcal} / \mathrm{mol}$ and an update width parameter of $1.0 \AA$. Crystal structure prediction was conducted with the MS Polymorph Predictor module. Crystal packings were generated randomly, energy minimized and subsequently ranked according to potential energy. For more details on Polymorph Predictor, the reader is referred to Gdanitz et al. [14] and Karfunkel et al. [15]. Amorphous polymer plasticizer and polymerplasticizer phases consisting of hydroxyl-terminated polybutadiene and dioctyl adipate were created using a combination of the algorithm developed by Theodorou and Suter [16] and the scanning method of Meirovitch [17] implemented in the MS Amorphous Phase module. A single bond was added per step, under the single substate per state rule, while using a substate width of 20 . A random number seed was used to insure a properly randomized distribution in the cell. Model systems thus built were submitted to a preliminary equilibration treatment consisting of 1000 steps using the canonical ensemble NVT (constant number of atoms, constant volume and constant temperature) dynamics simulation with a velocity rescaling method to maintain a constant temperature, followed by 100000 steps of minimization. Energy was minimized using three methods. The first used was steepest descent up to a maximum derivative of $100 \mathrm{kcal} \mathrm{mol}^{-1}$, followed by the conjugate gradient method (using the Polak-Ribiere algorithm) down to maximum derivative of $10 \mathrm{kcal} \mathrm{mol}^{-1}$. The Newton method using Broyden-Fletcher-Goldfarb-Shanno (bfgs) approach (the maximum derivative is $0.001 \mathrm{kcal} \mathrm{mol}^{-1}$ ) was used last. Model building was followed by NVT dynamics simulation for $1.0 \mathrm{~ns}$. Temperature in all simulations was equilibrated with the Andersen algorithm, using a collision rate of 1.0. The velocity Verlet algorithm was used to integrate equations of motion with a 1.0 fs time step. Only one blend composition was investigated, HTPB-DOA, containing one molecule of DOA and one 6-repeat unit HTPB chain, blend composition being of $49 \mathrm{w} \%$ HTPB and $51 \mathrm{w} \%$ DOA. Initial densities used for model building were experimental values $0.900 \mathrm{~g} \mathrm{~cm}^{-3}$ for HTPB and $0.927 \mathrm{~g} \mathrm{~cm}^{-3}$ for DOA. The density of amorphous blend system HTPB-DOA was calculated as the weight average of pure substance densities: initial HTPB-DOA density was estimated as $0.929 \mathrm{~g} \mathrm{~cm}^{-3}$. The RDX-based PBX HTPBDOA composition was based on $83 \mathrm{w} \%$ RDX crystal phase (16 RDX molecules) and 17 w\% HTPB-DOA amorphous phase (6-repeat unit HTPB chains and one DOA molecule). Using the MS surface builder module, RDX surfaces were first prepared by cleaving the crystal phase at the desired surface plane $(h k l)$, while insuring that the width and depth of the surface are larger than the non-bonded cut-off distance of $10.8 \AA$. The cleaved surface was minimized as described above and was then placed in a supercell $(2 \times 2 \times 1)$, over which the HTPB-DOA amorphous phase was inserted. The $c$-axis of the supercell was extended to $30 \AA$, so that HTPB-DOA can 'see' only one side of the surface even under periodic boundary conditions. NVT molecular dynamics simulation was then performed for 200 ps with a 1.0 fs time step at $298 \mathrm{~K}$, 
followed by 50 ps production runs, during which data were collected for subsequent analysis.

\section{Results and Discussion}

In this work, the aim is to use modelling techniques for RDX-based PBXs HTPBDOA and investigate the effect of interface interactions on mechanical properties. To evaluate the usefulness of the approach for real systems, for which crystal structure data are often unavailable, the RDX crystal structure was also predicted.

\subsection{Crystal Structure Prediction}

The optimized crystal parameters are reported in Table 1. A large number of structures are generated and ranked from the lowest total potential energy $E_{p}$ to the highest. The lowest-energy corresponds to an orthorhombic Pbca space group, whereas the second lowest energy is monoclinic $\mathrm{C} 2 / \mathrm{c}$. In the seven lowest energy structures generated, density fluctuates from 1.75 to $1.99 \mathrm{~g} \mathrm{~cm}^{-3}$. Large energy differences are observed, only the lowest being stable. Experimental data for the RDX crystal structure [18] is compared to the lowest-energy result in Table 2. RDX crystallizes in the orthorhombic space group, as found using Polymorph Predictor. Unit cell dimensions are in good agreement with experiment, $a, b$ and $c$ vector units showing discrepancies of $1.9 \%,-2.7 \%$ and $-4.7 \%$, respectively.

Table 1. Lowest-energy crystal structure models for RDX showing space group, density $\rho$ $\left(\mathrm{g} / \mathrm{cm}^{3}\right)$, potential energy $\mathrm{E}_{\mathrm{p}}(\mathrm{kcal} / \mathrm{mol})$, cell vectors a, b and $\mathrm{c}(\AA)$ and angles $\alpha, \beta$ and $\gamma\left({ }^{\circ}\right)$

\begin{tabular}{lcccccccc}
\hline $\begin{array}{l}\text { Space } \\
\text { group }\end{array}$ & $\rho$ & $\mathrm{E}_{\mathrm{p}}$ & $\mathrm{a}$ & $\mathrm{b}$ & $\mathrm{c}$ & $\alpha$ & $\beta$ & $\gamma$ \\
\hline Pbca & 1.91 & -1611 & 13.43 & 11.26 & 10.21 & 90.00 & 90.00 & 90.00 \\
$\mathrm{C} 2 / \mathrm{c}$ & 1.86 & -1602 & 24.73 & 5.93 & 27.30 & 90.00 & 156.58 & 90.00 \\
$\mathrm{Pnma}$ & 1.75 & -1592 & 10.66 & 26.05 & 6.07 & 90.00 & 90.00 & 90.00 \\
$\mathrm{P} 2_{1} 2_{1} 2_{1}$ & 1.90 & -806 & 5.87 & 15.38 & 8.59 & 90.00 & 90.00 & 90.00 \\
$\mathrm{P} 2_{1} / \mathrm{c}$ & 1.89 & -806 & 6.62 & 11.20 & 10.63 & 90.00 & 83.33 & 90.00 \\
$\mathrm{Pna} 2$ & 1.99 & -798 & 7.21 & 10.85 & 9.46 & 90.00 & 90.00 & 90.00 \\
$\mathrm{P} 2_{1}$ & 1.99 & -399 & 6.51 & 9.46 & 6.51 & 90.00 & 67.24 & 90.00 \\
\hline
\end{tabular}

The predicted density is $5.7 \%$ higher, which is reasonable considering the approximations made when using a force field. This excellent match clearly indicates that Polymorph Predictor, coupled to the COMPASS force field, is adequate to predict crystal structures of molecules representing similar chemical and conformational properties. The force field used should however always be evaluated for the chemical class under investigation. Crystal structures of molecules of higher flexibility will be increasingly difficult to predict, and therefore this approach can only be used, at the present time, for rigid molecules. For more flexible molecules, determining the experimental crystal structure is still essential. 
Table 2. Properties of the RDX crystal: density $\left(\mathrm{g} / \mathrm{cm}^{3}\right)$ and cell vectors $(\AA)\left(\alpha=\beta=\gamma=90^{\circ}\right)$

\begin{tabular}{lcccc}
\hline RDX (Pbca) & $\rho$ & $\mathrm{a}$ & $\mathrm{b}$ & $\mathrm{c}$ \\
\hline Experimental $^{[18]}$ & 1.82 & 13.18 & 11.57 & 10.71 \\
Predicted & 1.91 & 13.43 & 11.26 & 10.21 \\
\hline
\end{tabular}

\subsection{Simulation of the Crystalline RDX-Amorphous HTPB-DOA Supersystem}

In the following simulation step, HTPB, DOA and HTPB-DOA amorphous phase models were built and optimized. HTPB-DOA models were selected using a criteria of low energy. This step was followed by construction of the crystalline RDXamorphous HTPB-DOA supersystem, which is the most crucial step of this study, as it will allow interfacial interactions to be investigated. Further, mechanical properties will be estimated for an ideal 50:50 HTPB-DOA blend, for which no experimental mechanical properties have been reported in the literature. Similar modelling techniques have been used previously by various groups to simulate interactions in various PBXs systems [11]. It has been shown [8, 9] that DOA acts as a plasticizer in PBX formulations.

In the present work, an amorphous phase containing the polymer and the plasticiser was built using a concentration used in experimental formulations. It was however not possible to maintain a reasonable system size while strictly respecting the relative concentrations of amorphous and crystalline material. Instead, focus was put, when selecting the crystal-amorphous phase composition, on choosing a size which allowed reproduction of surface interactions with the plasticizer. Actual formulations are slightly richer in crystal phase than in plasticizer-polymer binding amorphous phase (90\% crystal phase versus the modelled 83\%). The simulated HTPB-DOA-RDX system is depicted in Fig. 2 for the three crystal surface planes considered. In the initial amorphous phase, HTPB and DOA were in a random coil-like conformation. This is clearly not the case after energy minimisation in presence of RDX: for all crystal cell orientations, HTPB and DOA molecules are now relatively extended, DOA is at the left in all three packing arrangements, and HTPB at the right side in the cell. Both are lying almost flat on top of the crystal structure, although some kinks are observable in both molecules above the (210) crystal cell. These chain arrangements are believed to allow a minimum in energy through intermolecular interactions. However, the occurrence of kinks in the (210) case qualitatively indicates that RDX/DOA and $\mathrm{RDX} / \mathrm{HTPB}$ interactions are weaker in this case. Although DOA mainly acts as a HTPB plasticizer, due to the small concentration of the HTPB-DOA mixture both in the modelled systems $(17 \mathrm{w} \%)$ and in typical experimental formulations (around $10 \%)$, DOA inevitably comes in close contact with the RDX crystalline phase and also forms strong van der Waals and electrostatic interactions. 

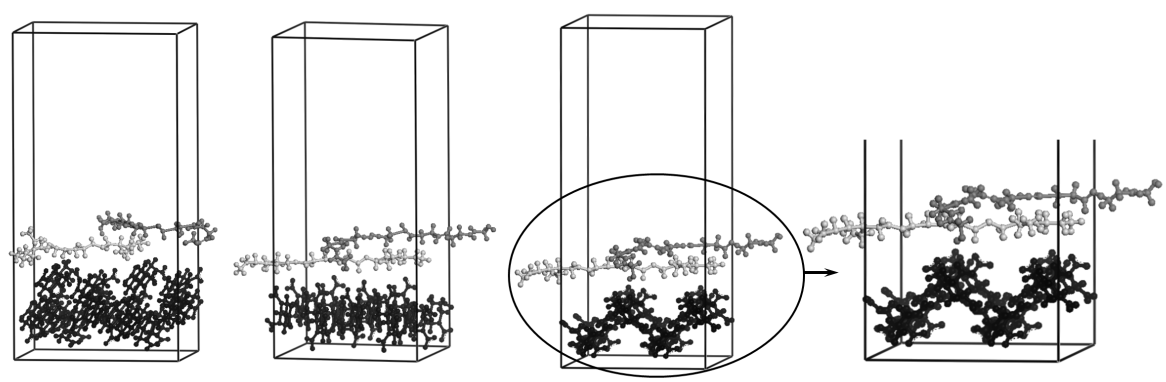

Fig. 2. Typical simulated HTPB-DOA/RDX supercells with (210) (left), (200) (center) and (020) (right) crystal surface, respectively, and zoom of the HTPB-DOA/RDX supercells with (020) crystal surface, (HTPB in gray, DOA in light gray and RDX in black)

Stability was checked by following potential and nonbond energy fluctuations. Systems were taken as stable and used for further calculations when these did not reach $10 \%$. The interaction energy was calculated by using the following equation.

$$
E_{\text {Interaction }}=E_{\text {total }}-\left(E_{\text {sulface }}+E_{\text {polymer }}\right)
$$

$E_{\text {total }}$ is the energy of the surface and the polymer, $E_{\text {surface }}$ is the energy of the surface without the polymer and $E_{\text {polymer }}$ is the energy of the polymer without the surface. The binding energy $E_{\text {binding }}$ reflects the intermolecular interactions between polymers and crystal, which is defined as the negative value of the interaction energy, which can be written as $E_{\text {binding }}=-E_{\text {interaction }}$. Results, reported in Table 3, vary with the crystallographic surface that is in contact with the polymer-plasticizer mixture. The (020) surface of RDX has the largest binding energies and therefore strongest ability to interact with the polymer, and the (210) surface has the smallest.

Table 3. Average binding energy (in $\mathrm{kcal} / \mathrm{mol}$ ) for different crystalline planes

\begin{tabular}{lc}
\hline$(h k l)$ plane & $\mathrm{E}_{\text {binding }}(\mathrm{kcal} / \mathrm{mol})$ \\
\hline$(020)$ & 64 \\
$(200)$ & 53 \\
$(210)$ & 41 \\
\hline
\end{tabular}

Pair correlation function $\mathrm{g}(\mathrm{r})$ was used to verify more objectively the degree of organization in these models [19]. Figure 3 shows $g(r)$ values obtained after 250 ps NVT simulation of the three models. The three surfaces have comparable $g(r)$ values at first glance, although maximum $\mathrm{g}(\mathrm{r})$ values are obtained for $(020)$ and (200) surfaces, confirming the conclusion obtained from binding energies. In all cases, the largest peaks appear at $\mathrm{r}$ distances below $3.5 \AA$. For interatomic $\mathrm{r}$ distances higher than $3.5 \AA$, very few peaks are observed, and those which are have very small intensities, indicating that long-range order due to atom interactions is weak. These observations confirm that interactions at short distance play a role in system stability. 

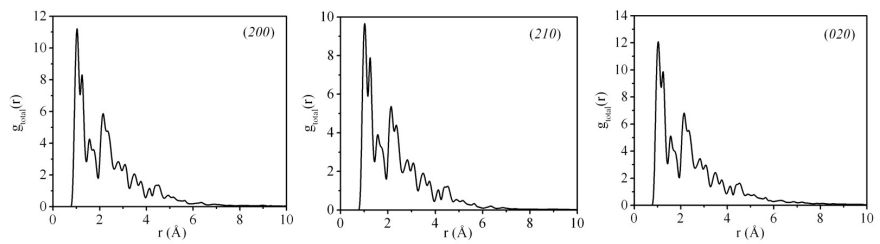

Fig. 3. Pair correlations functions total $g(r)$ calculated for all atoms in HTPB-DOA/RDX models for different $(h k l)$ planes, after 250 ps NVT dynamics simulation

Figure 4 shows the pair correlation function $\mathrm{g}(\mathrm{r})$ between $\mathrm{H}$ atoms of terminal HTPB hydroxyls and nitro oxygen atoms of RDX. The smallest distance d between $\mathrm{H}$ and $\mathrm{O}$ is observed for the HTPB-DOA/RDX $(020)$ surface $(\mathrm{d}=1.75 \AA)$, which also exhibits the most intense $\mathrm{g}(\mathrm{r})$ peak, indicative of stronger and more numerous van der Waals and electrostatic interactions. This is in good correlation with the highest binding energy value observed for this model. In the case of the (200) and (210) crystallographic planes, peak distances vary between $2.05 \AA$ and $2.15 \AA$ respectively. Therefore, when HTPB comes in contact with a RDX crystal, the strongest interactions are observed with the (020) plane, and maximising the surface of this plane should improve adhesion between the crystalline phase and the polymer binder.

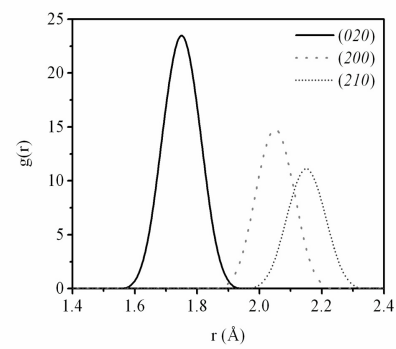

Fig. 4. Pair correlation function $\mathrm{g}(\mathrm{r})$ for $\mathrm{H}$ atom pairs of terminal HTPB hydroxyls and $\mathrm{O}$ atoms in RDX nitro groups for different $(h k l)$ planes

\subsection{Mechanical Properties}

NPT (constant number of atoms, constant pressure and constant temperature) dynamics simulations were finally applied to these simulated systems to investigate mechanical properties of RDX-based PBXs. In this case the $c$-axis of the supercell is not extended. Calculations are based on a formalism due to Parrinello and Rahman [20] in which the elastic stiffness tensor $C_{i j}$ is expressed in terms of fluctuations in the elastic strain tensor $\left\langle\varepsilon_{i} \varepsilon_{j}\right\rangle$ for the material. The NPT dynamics simulation was then performed for 200 ps with a time step of $1.0 \mathrm{fs}$ at $298 \mathrm{~K}$ and a cell mass of 20.00 atomic mass units, followed by production runs of $100 \mathrm{ps,} \mathrm{during} \mathrm{which} \mathrm{data} \mathrm{were} \mathrm{collected} \mathrm{for}$ subsequent analysis. In the theory of linear elasticity, the stress and strain tensors $\sigma$ and $\varepsilon$ are related to the elastic stiffness tensor $C_{i j}$ by $\sigma_{i}=C_{i j} \times \varepsilon_{j}(i, j=1,2, \ldots, 6)[21]$. 
For an isotropic material, the stiffness matrix may be described by specifying only Lamé coefficients $\lambda$ and $\mu$.Poisson's ratio $v$ and various moduli (Young's $E$, bulk $K$, shear $G$ ) can be calculated assuming the material to be isotropic. Elastic moduli may then be written in terms of the Lamé coefficients as follows [22]:

$$
v=\frac{\lambda}{2(\lambda+\mu)}, \quad E=\mu\left(\frac{3 \lambda+2 \mu}{\lambda+\mu}\right), \quad K=\lambda+\frac{2}{3} \mu, \quad G=\mu
$$

Poisson's ratio $v$ is the ratio of transverse contraction strain to longitudinal extension strain in the direction of the stretching force. Tensile modulus $E$ is also known as the Young modulus, and is the tangent or secant modulus of elasticity of a material in tension. The bulk modulus $(K)$ of a substance measures the resistance of the substance to uniform compression. It is defined as the pressure increase needed to cause a given relative decrease in volume. The shear modulus $(G)$ is defined as the ratio of shear stress to the shear strain. The $K / G$ indicates the extent of the plastic range, this ratio is associated with ductility in cases where $K / G$ is high, and with brittleness when it is low. Mechanical properties thus estimated should be taken as approximations, due to the small system size and anisotropy as well as force field approximations, and should be used for comparison purposes only. Values are summarized in Table 4.

Table 4. Mechanical properties of RDX-Based PBXs (in GPa) for the various ( $h k l$ ) surfaces

\begin{tabular}{lccccc}
\hline & $(200)$ & $(210)$ & $(020)$ & RDX & Ref \\
\hline Tensile modulus $(E)$ & 23 & 68 & 11 & 18 & {$[23]$} \\
Poisson ratio $(v)$ & 0.33 & 0.32 & 0.36 & 0.22 & {$[23]$} \\
Bulk Modulus $(K)$ & 23 & 65 & 14 & 13 & {$[24]$} \\
Shear modulus $(G)$ & 8.5 & 26 & 4 & - & \\
K/G & 2.7 & 2.6 & 3.5 & - & \\
\hline
\end{tabular}

In Table 4, Poisson ratios are similar for all three models, but a $22 \%$ difference in modulus is noted compared to the experimental value reported for the RDX crystal, which confirms the semi-quantitative nature of properties estimated using this approach. All other calculated mechanical properties vary with $(h k l)$ directions: tensile, bulk and shear moduli are lowest for $(020)$ and highest for (210). Resistance to elastic deformation, expressed by the tensile modulus $(E)$, has decreased for the $(020)$ plane, indicating less rigidity in contrast to (200) and (210) planes. In other words, the HTPB-DOA/RDX (020) blend behaves more like a rubber and is more flexible. Finally, one of the most important parameters for explosive formulation is the $K / G$ quotient, which is a measure of brittleness. It is highest for $(020)$ and lower for (210) and (200), showing that ductility is better in the latter two cases. Interactions are less important, which explains the increased ductility. 


\section{Conclusions}

The HTPB-DOA/RDX energetic blend system has been used to investigate the usefulness of atomistic modelling in the study and design of novel energetic materials. With the rigid RDX molecule, it was possible to predict, using the Polymorph Predictor module, the correct crystal space group, while unit cell dimensions and density showed a good match. This prediction approach may therefore be used for new energetic molecules for which no experimental data are available. Interactions between a crystalline, energetic molecule (RDX), a polymer binder (HTPB) and a plasticizer (DOA) were modelled under periodic boundary conditions following an approach previously proposed in the literature for crystalline-polymer interfaces [11]. The most favourable interactions occur between HTPB-DOA and the RDX (020) crystal face, and $\mathrm{g}(\mathrm{r})$ calculations showed that the main interaction at play was hydrogen bonding between the terminal hydroxyl group of HTPB and RDX oxygen atoms of nitro groups. It can be concluded that crystal morphology will have an effect on mechanical properties of the final blend systems, and that improvement in mechanical properties could be obtained by adjusting crystal growth conditions to favor large $(020)$ crystal surfaces. Finally, Parrinello-Rahman calculations of isotropic moduli (Young's E, bulk $K$, shear $G$ ) for crystalline RDX-based PBXs show that the resistance to elastic deformation is decreased in the case of (020) as compared to (210) and (200). Hydrogen bonds and other interactions that occur most dominantly for the RDX (020) crystal face with HTPB-DOA therefore provide a more flexible mixture, lowering the modulus and increasing ductility. This result suggests that choosing appropriate conditions (solvent, temperature) to favor the growth of (020) crystal faces may improve mechanical properties of resulting PBX formulations. These properties will be explored in the future using experimental methods in our lab.

Acknowledgments. The authors gratefully thank scientists P. Lessard and P. Brousseau from DRDC Valcartier for the fruitful discussions on all experimental data of polymer and plasticizers used in this work. The authors also wish to acknowledge the support of the Centre de Bioinformatique et de Biologie Computationelle of Université Laval. Financial support for this work was provided by the NSERCC (National Science and Engineering Council of Canada).

\section{References}

1. Dagley, I.J., Parker, R.P., Jones, D.A., Montelli, L.: Simulation and Moderation of the Thermal Response of Confined Pressed Explosive Compositions. Combust. Flame. 106, 428-441 (1996)

2. Scholtes, J.H.G.: Onderzoek Naar de Variatie van Mechanische Eigenschappen van HTPB PBX's. TNO-PML 1997-A55 report (1997)

3. Yoo, C.-S., Cynn, H., Howard, W.M., Holmes, N.: Equations of State of Unreacted High Explosives at High Pressures. In: 11th International Detonation Symposium Snowmass Village, CO, USA (1998)

4. Provatas, A.: Formulation and Performance Studies of Polymer Bonded Explosives (PBX) Containing Energetic Binder Systems. Part 1, Technical Report DSTO-TR-1397 (2003) 
5. Siviour, C.R., Gifford, M.J., Walley, S.M., Proud, W.G., Field, J.E.: Particle Size Effects on the Mechanical Properties of a Polymer Bonded Explosive. J. Mater. Sci. 39, 1255$1258(2004)$

6. Doherty, R.M., Watt, D.S.: Relationship between RDX Properties and Sensitivity. Propellants, Explos. Pyrotech. 33, 4-13 (2008)

7. Sewell, T.D., Menikoff, R., Bedrov, D., Smith, G.D.: A Molecular Dynamics Simulation Study of Elastic Properties of HMX. J. Chem. Phys. 119, 7417-7426 (2003)

8. Jaidann, M., Abou-Rachid, H., Lafleur-Lambert, X., Lussier, L.-S., Gagnon, N., Brisson, J.: Modeling and Measurement of Glass Transition Temperatures of Energetic and Inert Systems. Polym. Eng. Sci. 48, 1141-1150 (2008)

9. Abou-Rachid, H., Lussier, L.-S., Ringuette, S., Lafleur-Lambert, X., Jaidann, M., Brisson, J.: On the Correlation between Miscibility and Solubility Properties of Energetic Plasticizers/Polymer Blends: Modeling and Simulation Studies. Propellants, Explos. Pyrotech. 33, 301-310 (2008)

10. ter Horst, J.H., Geertman, R.M., van der Heijden, A.E., van Rosmalen, G.M.: The Influence of a Solvent on the Crystal Morphology of RDX. J. Cryst. Growth. 198, 773-779 (1999)

11. Xiao, J., Huang, H., Li, J., Zhang, H., Zhu, W., Xiao, H.: A Molecular Dynamics Study of Interface Interactions and Mechanical Properties of HMX-based PBXs with PEG and HTPB. THEOCHEM. 851, 242-248 (2008)

12. Zhu, W., Xiao, J., Zhu, W., Xiao, H.: Molecular Dynamics Simulations of RDX and RDXBased Plastic-Bonded Explosives. J. Hazard. Mater (2008), doi:10.1016/j.jhazmat.2008.09.021

13. Sun, H.: COMPASS: An ab Initio Force-Field Optimized for Condensed-Phase Applications-Overview with details on Alkane and Benzene Compounds. J. Phys. Chem. B. 102, 7338-7364 (1998)

14. Gdanitz, R.J., Karfunkel, H.R., Leusen, F.J.J.: The Prediction of Yet-Unknown Molecular Crystal Structures by Solving the Packing Problem. J. Mol. Graph. 11, 275-276 (1993)

15. Karfunkel, H.R., Rohde, B., Leusen, F.J.J., Gdanitz, R.J., Rihs, G.: Continuous Similarity Measure between Nonoverlapping X-ray Powder Diagrams of Different Crystal Modifications. J. Comput. Chem. 14, 1125-1135 (1993)

16. Theodorou, D.N., Suter, U.W.: Atomistic Modeling of Mechanical Properties of Polymeric Glasses. Macromolecules 19, 139-154 (1986)

17. Meirovitch, H.: Computer Simulation of Self-Avoiding Walks: Testing the Scanning Method. J. Chem. Phys. 79, 502-508 (1983)

18. Choi, C.S., Prince, E.: The Crystal Structure of Cyclotrimethylenetrinitramine. Acta Crystallogr. Sect. B. 28, 2857-2862 (1972)

19. Hansen, J.-P., McDonald, I.R.: Theory of Simple Liquids, 2nd edn. Academic Press, London (1990)

20. Parrinello, M., Rahman, A.: Strain Fluctuations and Elastic Constants. J. Chem. Phys. 76, 2662-2666 (1982)

21. Weiner, J.H.: Statistical Mechanics of Elasticity. John Wiley, New York (1983)

22. Mavko, G., Mukerji, T., Dvorkin, J.: The Rock Physics Handbook. Cambridge University, Cambridge (2003)

23. Annapragada, S.R., Sun, D., Garimella, S.V.: Prediction of Effective Thermo-Mechanical Properties of Particulate Composites. Comp. Mat. Sci. 40, 255-256 (2007)

24. Sewell, T.D., Bennett, C.M.: Monte Carlo Calculations of the Elastic Moduli and PressureVolume-Temperature Equation of State for Hexahydro-1,3,5-trinitro-1,3,5-triazine. J. Appl. Phys. 88, 88-95 (2000) 